\title{
Theoretical studies of the first-row transition metal phosphides
}

\author{
Glenna S. M. Tong \\ Department of Chemistry, The University of Hong Kong, Hong Kong, China
}

G. H. Jeung

Laboratorie de Chimie Théorique (CNRS UMR6517), Case 521, Campus de St-Jéröme, Université de Provence, 13397 Marseille Cedex 20, France

\author{
A. S-C. Cheunga) \\ Department of Chemistry, The University of Hong Kong, Hong Kong, China
}

(Received 25 November 2002; accepted 25 February 2003)

\begin{abstract}
The electronic structures and spectroscopic parameters of the ground and some low-lying excited states of the first-row transition metal phosphides have been calculated with the density functional theory using the Becke three-parameter hybrid exchange functional with the Lee-Yang-Parr correlation functional (B3LYP). The ground states of the transition metal phosphides are found to be ${ }^{1} \Sigma^{+}$(ScP), ${ }^{2} \Delta$ (TiP), ${ }^{3} \Delta$ (VP), ${ }^{4} \Sigma^{-}$(CrP), ${ }^{5} \Pi$ (MnP), ${ }^{6} \Sigma^{+}$(FeP), ${ }^{5} \Delta$ (CoP), ${ }^{4} \Delta$ (NiP), and ${ }^{3} \Sigma^{-}(\mathrm{CuP})$. The B3LYP functional predicts an increase in covalent character in the bonds between the metal and the phosphorus across the transition metal series. The energies of the low-lying excited states relative to the ground state for TiP, FeP, and CoP have been found to be so small that many low-lying states are possible candidates to be the ground state. (C) 2003 American Institute of Physics. [DOI: 10.1063/1.1568078]
\end{abstract}

\section{INTRODUCTION}

Understanding the nature of bonding between a transition metal (TM) and a main group element is of interest in many areas of science, such as surface science, ${ }^{1}$ catalysis, ${ }^{2}$ astrophysics, ${ }^{3,4}$ and organometallic chemistry. ${ }^{5,6}$ Amongst these compounds, the transition metal oxides, ${ }^{7}$ halides, ${ }^{8}$ and nitrides $^{9}$ are the most studied and well characterized. However, despite of the fact that transition metal phosphides (TMP) possess remarkable properties that promise potential applications in semiconductors, luminescent devices and electronic components, ${ }^{10}$ they are rarely studied. Theoretical studies of TM systems are challenging due to the near degeneracy and the strong dynamical correlation effects of the $d$ electrons. ${ }^{11}$ High level $a b$ initio methods like the multireference configuration interaction (MRCI) would normally be required to properly describe the chemical bonding involving $d$ electrons. However, such calculations are usually time consuming and computationally demanding, and have so far been performed on $\mathrm{ScP}^{12}$ and $\mathrm{TiP}^{13}$ molecules only. Recently, there was a report of the experimental observation and analysis of photoelectron spectra of some Group III phosphides. ${ }^{14}$ However, to the best of our knowledge, experimental spectroscopic work has not been performed to any of the TMP.

Density functional theory (DFT) has been quite successful in explaining and predicting behavior and properties of a wide variety of chemical systems and attracting much attention of theoretical chemists. DFT has the advantage of providing quite accurate estimates with a much faster speed and a much-reduced basis set requirement when compared with traditional correlation techniques. The performance of a DFT

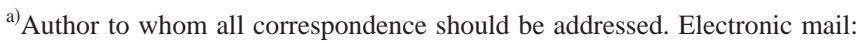
hrsccsc@hku.hk
}

calculation depends critically on the choice of the functionals. One of the most popular and widely used DFT functionals, developed by Becke, called B3LYP, ${ }^{15,16}$ shows very promising results for transition metal systems. This hybrid functional has been used in our recent calculation to obtain bond lengths, vibrational frequencies, and bond energies of alkali metal-transition metal diatomic systems, and the results are satisfactory. ${ }^{17}$ This hybrid functional includes a mixture of a traditional Hartree-Fock-like exchange energy, the Slater exchange functional, with gradient corrections due to Becke, and the correlation potential of Vosko, Wilk, and Nusair, with gradient corrections due to Lee, Yang, and Parr. ${ }^{15,16}$

In this work, we report DFT study of the ground and some low-lying excited states of the first-row TMP using the B3LYP functional. Equilibrium bond length, $r_{e}$, electronic term energy, $T_{e}$, harmonic vibrational frequency, $\omega_{e}$, dipole moment, $\mu_{e}$, and dissociation energy, $D_{e}$, of the nine firstrow TMP molecules were calculated. The chemical bonding of these phosphide molecules has also been examined. It is hoped that our computational results will stimulate experimental studies of these TMP molecules.

\section{COMPUTATIONAL METHODS}

The basis set used in our calculations for the first-row transition metal atoms consists of the $14 s, 11 p, 6 d$, and $3 f$ primitive Gaussian functions constructed by augmenting Wachters' $14 s, 9 p, 5 d$ basis with two additional diffuse $p$ functions to describe the $4 p$ orbitals and an extra $d$ function as suggested by Hay, and three primitive $f$ functions. The primitive functions were contracted to $8 s, 6 p, 4 d$, $1 f$ (Wachters $+\mathrm{f} \mathrm{set}$ ). ${ }^{18}$ For $\mathrm{Sc}$ and $\mathrm{Ti}$ atoms, in order to allow for $3 p$ orbital correlations, the $p$ contraction is 
TABLE I. Calculated equilibrium bond lengths $\left(r_{e}\right.$, in $\AA$ ), dissociation energies $\left(D_{e}\right.$, in $\left.\mathrm{eV}\right)$, harmonic vibrational frequencies $\left(\omega_{e}\right.$, in $\left.\mathrm{cm}^{-1}\right)$, effective nuclear charges $\left(Z_{\text {eff }}\right)$, and dipole moments $\left(\mu_{e}\right.$, Debye) of the ground state first-row TM phosphides using B3LYP method.

\begin{tabular}{lcccccc}
\hline \hline & State & $r_{e}$ & $D_{e}{ }^{\mathrm{a}}$ & $\omega_{e}$ & $\mu_{e}$ & $Z_{\text {eff }}$ \\
\hline $\mathrm{ScP}$ & ${ }^{1} \Sigma^{+}$ & 2.173 & $2.77 / 3.55$ & 537 & 6.83 & 0.66 \\
$\mathrm{TiP}$ & ${ }^{2} \Delta$ & 2.139 & $2.67 / 2.90$ & 454 & 6.35 & 0.62 \\
$\mathrm{VP}$ & ${ }^{3} \Delta$ & 2.140 & $3.26 / 4.10$ & 390 & 4.24 & 0.42 \\
$\mathrm{CrP}$ & ${ }^{4} \Sigma^{-}$ & 2.200 & $3.63 / 2.21$ & 351 & 4.02 & 0.38 \\
$\mathrm{MnP}$ & ${ }^{5} \Pi$ & 2.158 & $1.56 / \cdots{ }^{\mathrm{c}}$ & 406 & 4.40 & 0.43 \\
$\mathrm{FeP}$ & ${ }^{6} \Sigma^{+}$ & 2.106 & $2.07 / 2.59$ & 423 & 3.99 & 0.40 \\
$\mathrm{CoP}$ & ${ }^{5} \Delta$ & 2.125 & $2.29 / 2.23$ & 378 & 3.68 & 0.36 \\
$\mathrm{NiP}$ & ${ }^{4} \Delta$ & 2.127 & $2.77 / 2.32$ & 391 & 2.86 & 0.28 \\
$\mathrm{CuP}$ & ${ }^{3} \Sigma^{-}$ & 2.160 & $\cdots / 2.13^{\mathrm{c}}$ & 358 & 2.82 & 0.27 \\
\hline \hline
\end{tabular}

${ }^{a}$ The first value is relative to the metal atomic state $4 s^{2} 3 d^{n}$ and the second value is relative to the metal atomic state $4 s^{1} 3 d^{n+1}$.

${ }^{\mathrm{b}} Z_{\mathrm{eff}}=\mu_{e} / r_{e}$.

${ }^{c}$ Reordering the orbitals of the atomic state did not give other states of the same multiplicity. They all converged to the same state.

changed to (3311111). The basis set for P is the aug-cc-pVTZ basis set of Dunning and co-workers. ${ }^{18}$ It consists of a $16 s$, $10 p, 3 d$, and $2 f$ primitives contracted to $6 s, 5 p, 3 d, 2 f$. In all cases, unrestricted B3LYP calculations were performed using the GAUSSIAN 98 suite of programs. ${ }^{19}$ An HF/6-31G** was first performed to generate an initial guess orbital. This was then followed by B3LYP calculations with the extended basis sets. Spectroscopic properties for different point group symmetry states for a given spin quantum number were then obtained by reordering the orbitals. Dissociation energy $D_{e}$ was computed as the difference in the total energies $E_{\text {tot }}$ of the TMP and its constituent atoms,

$$
D_{e}(\mathrm{TMP})=E_{\mathrm{tot}}(\mathrm{TM})+E_{\mathrm{tot}}(\mathrm{P})-E_{\mathrm{tot}}(\mathrm{TMP}) .
$$

The molecular states of the TMP were assigned using the Kohn-Sham (KS) orbitals. It has been argued that KS orbitals are only auxiliary functions and bear no physical signifi- cance and thus, they should not be used to assign molecular states. However, in recent publications, it has been shown that results obtained from DFT/KS orbitals are quite similar to the molecular orbitals obtained from ab initio methods, and that one can extract a lot of information about molecular systems from an analysis of their molecular orbitals even if DFT methods are used. ${ }^{20,21}$

\section{CHEMICAL BONDS OF THE GROUND STATE}

The calculated ground state spectroscopic parameters and population analysis of the first-row TMP are presented in Tables I and II, respectively. As seen from the population analysis and dipole moments, the bonding is suggested to contain both ionic and covalent contributions, where the covalent contribution increases across the period (with the exception of MnP that has a slight increase in dipole moment and net charge). The bonding arises from the interactions between the metal valence $3 d$ and $4 s$ orbitals and the phosphorus $3 p$ orbitals. The metal $\sigma$ orbitals undergo $s p_{\sigma}$ and $s d_{\sigma}$ hybridizations and interact with the phosphorus $3 p_{\sigma}$ orbital to form bonding, nonbonding, and antibonding orbitals. The metal $3 d_{\pi}$ orbitals interact with the $\mathrm{P} 3 p_{\pi}$ orbitals to give bonding and antibonding orbitals. The metal $3 d_{\delta}$ orbitals are nonbonding as there are no counterparts in phosphorous (P). Hence, the expected order of stability is $\sigma$ bond $>\pi$ bond $>\sigma$ and $\delta$ nonbonding $>\pi$ antibonding $>\sigma$ antibonding. However, the filling order is complicated by the fact that $d-d$ exchange energy is larger than the energy separation between different orbitals and the mixings of the metal $s^{2} d^{n}$ and $s^{1} d^{n+1}$ asymptotes. Moreover, as we move across the series, the energy of the metal valence orbitals ( $4 s$ and $3 d$ ) decrease so that it is higher than the $\mathrm{P} 3 p$ orbitals for the early TM atoms, but fall below that of $\mathrm{P} 3 p$ orbitals for latter TM atoms. ${ }^{22}$

TABLE II. Mulliken population analysis of the ground state of the first-row TM phosphides using the B3LYP method.

\begin{tabular}{|c|c|c|c|c|c|c|c|c|c|c|}
\hline & & $\begin{array}{c}\mathrm{ScP} \\
{ }^{1} \Sigma^{+}\end{array}$ & $\begin{array}{l}\text { TiP } \\
{ }^{2} \Delta\end{array}$ & $\begin{array}{l}\text { VP } \\
{ }^{3} \Delta\end{array}$ & $\begin{array}{c}\mathrm{CrP} \\
{ }^{4} \Sigma^{-}\end{array}$ & $\begin{array}{c}\mathrm{MnP} \\
{ }^{5} \Pi\end{array}$ & $\begin{array}{l}\mathrm{FeP} \\
{ }^{6} \Sigma^{+}\end{array}$ & $\begin{array}{c}\mathrm{CoP} \\
{ }^{5} \Delta\end{array}$ & $\begin{array}{l}\mathrm{NiP} \\
{ }^{4} \Delta\end{array}$ & $\begin{array}{l}\mathrm{CuP} \\
{ }^{3} \Sigma^{-}\end{array}$ \\
\hline \multirow[t]{12}{*}{$\mathrm{TM}$} & $s_{\sigma}$ & 0.58 & 0.50 & 0.84 & 0.81 & 0.99 & 0.95 & 0.86 & 1.01 & 1.12 \\
\hline & $p_{\sigma}$ & 0.05 & 0.04 & 0.06 & 0.06 & 0.08 & 0.08 & 0.09 & 0.09 & 0.10 \\
\hline & $p_{\pi x}$ & 0.07 & 0.06 & 0.03 & 0.02 & 0.17 & 0.10 & 0.07 & 0.05 & 0.04 \\
\hline & $p_{\pi y}$ & 0.07 & 0.06 & 0.03 & 0.02 & 0.03 & 0.10 & 0.07 & 0.05 & 0.04 \\
\hline & $d_{\sigma}$ & 0.32 & 0.37 & 0.91 & 0.94 & 1.08 & 1.08 & 1.09 & 1.87 & 1.92 \\
\hline & $d_{\pi x}$ & 0.67 & 0.74 & 0.89 & 0.94 & 1.26 & 1.76 & 1.86 & 1.92 & 1.95 \\
\hline & $d_{\pi y}$ & 0.67 & 0.74 & 0.89 & 0.94 & 1.13 & 1.76 & 1.86 & 1.92 & 1.95 \\
\hline & $d_{\delta+2}$ & 0.00 & 0.00 & 0.00 & 1.00 & 1.00 & 1.00 & 2.00 & 2.00 & 2.00 \\
\hline & $d_{\delta-2}$ & 0.00 & 1.00 & 1.00 & 1.00 & 1.00 & 1.00 & 1.00 & 1.00 & 2.00 \\
\hline & $4 s$ & 0.58 & 0.50 & 0.84 & 0.81 & 0.99 & 0.95 & 0.86 & 1.01 & 1.12 \\
\hline & $4 p$ & 0.19 & 0.15 & 0.12 & 0.10 & 0.27 & 0.28 & 0.22 & 0.19 & 0.17 \\
\hline & $3 d$ & 1.66 & 2.85 & 3.69 & 4.82 & 5.47 & 6.60 & 7.80 & 8.72 & 9.81 \\
\hline \multirow[t]{6}{*}{$\mathrm{P}$} & $s_{\sigma}$ & 1.89 & 1.92 & 1.94 & 1.92 & 1.86 & 1.85 & 1.86 & 1.88 & 1.80 \\
\hline & $p_{\sigma}$ & 1.15 & 1.16 & 1.23 & 1.26 & 0.98 & 1.03 & 1.09 & 1.13 & 1.05 \\
\hline & $p_{\pi x}$ & 1.22 & 1.17 & 1.06 & 1.02 & 1.55 & 1.11 & 1.06 & 1.01 & 1.00 \\
\hline & $p_{\pi y}$ & 1.22 & 1.17 & 1.06 & 1.02 & 0.82 & 1.11 & 1.06 & 1.01 & 1.00 \\
\hline & $3 s$ & 1.89 & 1.92 & 1.94 & 1.92 & 1.86 & 1.85 & 1.86 & 1.89 & 1.80 \\
\hline & $3 p$ & 3.59 & 3.50 & 3.34 & 3.30 & 3.35 & 3.25 & 3.21 & 3.16 & 3.06 \\
\hline$Q_{\mathrm{TM}}$ & & +0.56 & +0.49 & +0.35 & +0.27 & +0.27 & +0.17 & +0.12 & +0.08 & -0.10 \\
\hline
\end{tabular}




\section{A. Phosphides of the early TM: ScP, TiP, VP, and CrP}

The ground state of each TMP in the early period has a triple bond with the remaining valence electrons mainly localized on the TM. Accordingly, all valence electrons of Sc encumber the triple bond, giving rise to a ${ }^{1} \Sigma^{+}$ground state. The ground state of TiP is calculated to be ${ }^{2} \Delta$, with the fourth valence electron added to the $3 d_{\delta}$ orbital. In the MRCI-SD calculation by Harrison et al. ${ }^{13}$ the ground state was calculated to be ${ }^{2} \Sigma^{+}$, with the fourth valence electron going into the nonbonding $s d_{\sigma}$ orbital. In fact, these two states are calculated to have energy very close to each other. From MRCI-SD calculations of Harrison et al., ${ }^{13}$ the ${ }^{2} \Delta$ state is about $1700 \mathrm{~cm}^{-1}$ higher than the ${ }^{2} \Sigma^{+}$state and from our B3LYP calculations, the ${ }^{2} \Delta$ state is about $650 \mathrm{~cm}^{-1}$ lower than the ${ }^{2} \Sigma^{+}$state. It has been pointed out that the neglected multipolar terms in DFT methods may cause an overstabilization of the $4 s^{1} 3 d^{n+1}$ configuration. ${ }^{23-25}$ Since the ${ }^{2} \Delta$ state correlates to the atomic asymptote ${ }^{5} \mathrm{~F}\left(s^{1} d^{3}\right)$ whereas the ${ }^{2} \Sigma^{+}$state correlates to the atomic asymptote ${ }^{3} \mathrm{~F}$ $\left(s^{2} d^{2}\right)$, the overstabilization of the high-spin atomic state may change the order of the two states. However, MRCI method is also well known of its bias towards the low-spin $s^{2} d^{n}$ state. Experimental work could thus be valuable to confirm the ground state of TiP. The ground state of VP is ${ }^{3} \Delta$, with the fourth and the fifth valence electrons going into the metal nonbonding $s d_{\sigma}$ hybrid and the $3 d_{\delta}$ orbital. $\mathrm{CrP}$ has a ${ }^{4} \Sigma^{-}$ground state with the three unpaired electrons occupying the nonbonding $s d_{\sigma}$ hybrid and the two $3 d_{\delta}$ orbitals.

\section{B. Phosphides of the latter TM: MnP, FeP, CoP, NiP, and CuP}

If the bonding in MnP follows that of the early phosphides ( $\mathrm{ScP}$ to $\mathrm{CrP}$ ), the additional electron would go to the nonbonding $s d_{\sigma}$ hybrid or $3 d_{\delta}$ orbital, giving rise to the ${ }^{3} \Sigma^{-}$ or ${ }^{3} \Delta$ state as the ground state. Such bonding mechanism is actually not favorable as there is a loss in $d-d$ exchange energy and supplementary energy is required to polarize two electrons away from the $\mathrm{P}$ atom if the electron is added to the $s d_{\sigma}$ hybrid orbital. A better way may be to transfer one of the valence $\sigma$ or $\delta$ electrons of the ground state Mn to the $\mathrm{P} 3 p_{\pi}$ orbitals, leading to a ${ }^{5} \Pi$ state. The P $3 p_{\pi}$ orbitals then backdonate charges to the metal $3 d_{\pi}$ and $4 p_{\pi}$ orbitals (which are basically antibonding in character), increasing the $d-d$ exchange energy gain. The resultant $\sigma$ and $\pi$ bonds formed between $\mathrm{Mn}$ and $\mathrm{P}$ are polarized towards the phosphorus. Thus, a quintet state is more likely to be the ground state. Following the same bonding picture of $\mathrm{MnP}$, FeP would have its extra electron added to the $\mathrm{P} 3 p_{\pi}$ orbitals, mixed with $\mathrm{Fe}$ $3 d_{\pi}$ and $4 p_{\pi}$ orbitals, giving rise to a ${ }^{6} \Sigma^{+}$state as the ground state. For CoP, the ground state is calculated to be ${ }^{5} \Delta$ signifying that the extra electron goes into the nonbonding $3 d_{\delta}$ orbital. The addition of one more electron to the nonbonding $s d_{\sigma}$ hybrid in NiP gives a ${ }^{4} \Delta$ state as its ground state. Finally $\mathrm{CuP}$ would have its last electron occupying the nonbonding $3 d_{\delta}$ orbital leading to a ${ }^{3} \Sigma^{-}$ground state.

From Tables I and II, it is easily seen that the net charge on the metal atom and the dipole moment decrease across the period from the left-hand side $(\mathrm{Sc})$ to the right-hand side
TABLE III. Calculated bond lengths $\left(r_{e}\right.$, in $\AA$ ), excitation energies $\left(T_{e}\right.$, in $\left.\mathrm{cm}^{-1}\right)$, dissociation energies $\left(D_{e}\right.$, in $\left.\mathrm{eV}\right)$, harmonic vibrational frequencies $\left(\omega_{e}\right.$, in $\left.\mathrm{cm}^{-1}\right)$, effective nuclear charges $\left(Z_{\mathrm{eff}}\right)$, and dipole moments $\left(\mu_{e}\right.$, in Debye) of ScP for several electronic states using the B3LYP method. The values in parentheses are theoretical values from Tientega et al. (Ref. 12).

\begin{tabular}{|c|c|c|c|c|c|c|}
\hline State & $r_{e}$ & $T_{e}$ & $D_{e}{ }^{\mathrm{a}}$ & $\omega_{e}$ & $\mu_{e}$ & $Z_{\mathrm{eff}}^{\mathrm{b}}$ \\
\hline${ }^{1} \Sigma^{+}$ & $\begin{array}{c}2.173 \\
(2.277)\end{array}$ & $\begin{array}{c}0 \\
(0)\end{array}$ & $\begin{array}{c}2.77 / 3.55 \\
(1.55 / 3.25)\end{array}$ & $\begin{array}{c}537 \\
(446)\end{array}$ & 6.83 & $\begin{array}{c}0.66 \\
(0.23)\end{array}$ \\
\hline${ }^{1} \Pi$ & $\begin{array}{c}2.302 \\
(2.392)\end{array}$ & $\begin{array}{c}3049 \\
(4879)\end{array}$ & $\begin{array}{c}2.40 / 3.17 \\
(0.94)\end{array}$ & $\begin{array}{c}451 \\
(392)\end{array}$ & 5.32 & $\begin{array}{c}0.48 \\
(0.25)\end{array}$ \\
\hline${ }^{3} \Pi$ & $\begin{array}{c}2.298 \\
(2.375)\end{array}$ & $\begin{array}{c}3017 \\
(3364)\end{array}$ & $\begin{array}{c}2.40 / 3.18 \\
(1.12)\end{array}$ & $\begin{array}{l}458 \\
(397)\end{array}$ & 5.35 & $\begin{array}{c}0.49 \\
(0.23)\end{array}$ \\
\hline${ }^{3} \Phi$ & 2.386 & 7486 & $1.84 / 2.62$ & 408 & 7.27 & 0.64 \\
\hline${ }^{5} \Delta$ & 2.611 & 8496 & $1.72 / 2.49$ & 304 & 3.70 & 0.30 \\
\hline${ }^{5} \Pi$ & 2.655 & 11269 & $1.37 / 2.15$ & 284 & 3.46 & 0.27 \\
\hline
\end{tabular}

${ }^{a}$ The first value is relative to the atomic state $s^{2} d^{1}$ and the second value is relative to the atomic state $s^{1} d^{2}$.

${ }^{\mathrm{b}} Z_{\mathrm{eff}}=\mu_{e} / r_{e}$.

$(\mathrm{Cu})$ except a small rise at $\mathrm{Mn}$. Such trends are in accordance with the electronegativity difference between the metal and the phosphorus atoms, which also decreases across the series, and hence a decrease in ionic character across the series. It can also be seen that there is a sharp drop of dipole moment from TiP to VP and from CoP to NiP. This is due to the fact that electrons are added to the $s d_{\sigma}$ hybrid orbital sequentially, and these electrons are polarized away from the $\mathrm{P}$ atom and hence causing a decrease in the dipole moment.

\section{SPECTROSCOPIC PROPERTIES}

The ground and some low-lying excited states of the first-row TMP are discussed individually in the following sections. We obtained both the spectroscopic parameters and the Mulliken population analysis for the ground and some excited states of these nine TMP. Since the spectroscopic parameters are useful for experimentalists to search for these TMP and the population analysis of the ground states of these phosphides are important for the discussion, they are included in the text. However, tables concerning the population analysis of the excited states of individual molecules are available from the EPAPS. ${ }^{26}$

\section{A. ScP}

The spectroscopic parameters for the ground and some excited states of ScP are given in Table III. The ground state of ScP has a ${ }^{1} \Sigma^{+}$symmetry with a triple bond and a bond length of $2.173 \AA$. From the population analysis, it can be seen that both the $4 s$ and the $3 d_{\sigma}$ orbitals contribute to the $\sigma$ bond, with $4 s$ giving a larger contribution. This is in contrast with the nitride analogue, where the dominant contribution comes from the $3 d_{\sigma}$ orbital. This may come from the fact that $\mathrm{P} 3 p_{\sigma}$ orbital is more diffuse and therefore it has a better overlap with the diffuse metal $4 s$ orbital than the more compact metal $3 d$ orbital. Exciting the $\pi$-bonding electron from the ground state to the $s d_{\sigma}$ hybrid breaks the triple bond and gives rise to a ${ }^{1} \Pi$ state. This state lies $3049 \mathrm{~cm}^{-1}$ above the ground state. Triplet uncoupling of the excited $\sigma$ electron gives a ${ }^{3} \Pi$ state with adiabatic transition energy $T_{e}$ $=3017 \mathrm{~cm}^{-1}$. These two excited states have very similar 
spectroscopic parameters, as their major difference is the spin multiplicity. Triplet uncoupling of the $\pi$-bonding electron and exciting it from the ground state to the nonbonding $3 d_{\delta}$ orbital gives the ${ }^{3} \Phi$ state, which lies $7486 \mathrm{~cm}^{-1}$ above the ground state. Further excitation of the $\pi$-bonding electron from the ${ }^{3} \Pi$ state and quintet uncoupling to the $3 d_{\delta}$ and the essentially $3 d_{\pi}$ orbitals generated ${ }^{5} \Delta$ and ${ }^{5} \Pi$ states, respectively. These two states have a $\sigma$ bond, where the P $3 p_{\sigma}$ orbital not only makes a bonding with the $s d_{\sigma}$ hybrid orbital, but also with the $4 s p_{\sigma}$ hybrid orbital such that the $4 p$ population increases as can be seen in population analysis. This is because the $\pi$-bonding orbitals are now solely of $\mathrm{P} 3 p_{\pi}$ character so that there is no $3 d_{\pi}-3 p_{\pi}$ bonding to constraint the $4 s p_{\sigma}-3 p_{\sigma}$ bonding. Harrison and co-workers ${ }^{12}$ used MRCI-SD method to obtain spectroscopic parameters for $\mathrm{ScP}$ which gives the same energy ordering of states as ours: ${ }^{1} \Sigma^{+}<{ }^{3} \Pi<{ }^{1} \Pi$. Their calculated bond lengths are longer than our B3LYP values. This may be due to their selfconsistent-field calculations, which is biased in favor of the $s^{2} d^{1}$ asymptote, giving a larger contribution from the $4 s$ orbital (0.96) compared with our result (0.58). Their vibrational frequencies and dissociation energies are also calculated to be smaller than our values. Jeung ${ }^{27}$ has recently performed MRCI calculations on the ground state $\mathrm{ScP}$ with a more extended basis set and his calculated bond length, vibrational frequency, and dissociation energy (relative to the asymptotic products) are $2.248 \AA, 440 \mathrm{~cm}^{-1}$, and $3.81 \mathrm{eV}$, respectively. Our B3LYP results are in good agreements with Jeung's $a b$ initio data for $r_{e}$ and $D_{e}$ (within 7\% error), but not $\omega_{e}$ (more than $20 \%$ difference). Such discrepancy is also observed in the ground state calculations of $\mathrm{ScN},{ }^{27}$ where the B3LYP functional predicted a much larger $\omega_{e}$ compared with the MRCI and the experimental value. We have constructed the potential energy curve (PEC) for the ground state $\mathrm{ScN}$ and found that it led to a wrong asymptotic product, Sc $\left(3 d^{3}\right)$, which lies $4.2 \mathrm{eV}$ above the true asymptotic product, $\operatorname{Sc}\left(s^{1} d^{2}\right)$. This might mean that the B3LYP functional cannot properly mix these two atomic configurations. The same situation could happen to the $\mathrm{ScP}$ molecule.

\section{B. TiP}

The spectroscopic parameters for the ground and some excited states of TiP are presented in Table IV. The ground state of TiP is calculated to be of ${ }^{2} \Delta$ symmetry with a bond length $2.139 \AA$ A. It has a triple bond with the unpaired electron residing in the nonbonding metal-localized $3 d_{\delta}$ orbital. From the electron distribution in the population analysis, it is noted that as in the case of $\mathrm{ScP}$, both the $4 s$ and the $3 d_{\sigma}$ orbitals contribute to the $\sigma$ bond, with $4 s$ having a larger share. This is again due to the fact that metal $3 d$ orbitals are more compact and do not overlap with the diffuse P $3 p$ orbitals as good as the diffuse metal $4 s$ orbital. Excitation of the unpaired $\delta$ electron from the ${ }^{2} \Delta$ state to the $s d_{\sigma}$ hybrid gives rise to a ${ }^{2} \Sigma^{+}$state. This state lies only $647 \mathrm{~cm}^{-1}$ above the ${ }^{2} \Delta$ state and is thus also a possible candidate for TiP ground state. If the unpaired $3 d_{\delta}$ electron is promoted to the higher-lying $\pi$ orbital, it gives a ${ }^{2} \Pi$ state, which is $9158 \mathrm{~cm}^{-1}$ above the ground state. The higher-lying $\pi$ orbital in this state has contributions not only from $3 d_{\pi}-3 p_{\pi}$
TABLE IV. Calculated bond lengths $\left(r_{e}\right.$, in $\AA$ ), excitation energies $\left(T_{e}\right.$, in $\left.\mathrm{cm}^{-1}\right)$, dissociation energies $\left(D_{e}\right.$, in $\left.\mathrm{eV}\right)$, harmonic vibrational frequencies $\left(\omega_{e}\right.$, in $\left.\mathrm{cm}^{-1}\right)$, effective nuclear charges $\left(Z_{\text {eff }}\right)$, and dipole moments $\left(\mu_{e}\right.$, in Debye) of TiP for several electronic states using the B3LYP method. The values in parentheses are theoretical values from Glezakon et al. (Ref. 13).

\begin{tabular}{lcccccc}
\hline \hline State & $r_{e}$ & $T_{e}$ & $D_{e}{ }^{\mathrm{a}}$ & $\omega_{e}$ & $\mu_{e}$ & $Z_{\text {eff }}{ }^{\mathrm{b}}$ \\
\hline${ }^{2} \Delta$ & 2.139 & 0 & $2.67 / 2.90$ & 454 & 6.35 & 0.62 \\
& $(2.217)$ & $(1690)$ & $(1.82)$ & $(434)$ & $(7.2)$ & $(0.45)$ \\
${ }^{2} \Sigma^{+}$ & 2.078 & 647 & $2.59 / 2.82$ & 483 & 4.55 & 0.46 \\
& $(2.158)$ & $(0)$ & $(2.04)$ & $(465)$ & $(4.4)$ & $(0.48)$ \\
${ }^{2} \Phi$ & 2.271 & 2043 & $2.42 / 2.64$ & 404 & 4.88 & 0.45 \\
${ }^{2} \Pi$ & 2.168 & 9158 & $1.53 / 1.76$ & 385 & 5.71 & 0.55 \\
& $(2.280)$ & $(9900)$ & $(0.78)$ & $(343)$ & $(5.3)$ & $(0.41)$ \\
${ }^{4} \Phi$ & 2.239 & 1970 & $2.43 / 2.65$ & 454 & 5.04 & 0.47 \\
${ }^{6} \Pi$ & 2.553 & 8145 & $1.66 / 1.89$ & 307 & 3.73 & 0.31 \\
\hline \hline
\end{tabular}

${ }^{a}$ The first value is relative to the atomic state $s^{2} d^{2}$ and the second value is relative to the atomic state $s^{1} d^{3}$.

${ }^{\mathrm{b}} Z_{\mathrm{eff}}=\mu_{e} / r_{e}$.

overlap, but also $4 p_{\pi}-3 p_{\pi}$ overlap. This is because excitation to the $3 d_{\pi}$ orbital reduces $\mathrm{P}$ to Ti backdonation, which decreases the covalent contribution, and hence an increase in the net charge. To minimize the loss in covalent bonding, the $\pi$ orbital becomes a mixture of $3 d_{\pi}$ and $4 p_{\pi}$. Since the $4 p_{\pi}$ orbital is in a different region of space, it does not interfere with the $\mathrm{P}$ to Ti backdonation. Therefore, the ${ }^{2} \Pi$ state shows an increase in the net charge and an increase in the $4 p_{\pi}$ population relative to the ${ }^{2} \Delta$ ground state. Exciting the $\pi$-bonding electron from the ${ }^{2} \Delta$ state to the nonbonding $s d_{\sigma}$ hybrid results in ${ }^{2} \Phi$ and ${ }^{4} \Phi$ states, which are, respectively, 2043 and $1970 \mathrm{~cm}^{-1}$ above the ${ }^{2} \Delta$ state. These two states have very similar spectroscopic parameters since their main difference comes from the spin multiplicity. As in ScP, the higher spin state is lower in energy, which conforms to the Hund's rule. Further excitation of another $\pi$-bonding electron from the ${ }^{4} \Phi$ state to the higher-lying $\pi$ orbital and uncoupling the electron will give rise to a ${ }^{6} \Pi$ state, which is $8145 \mathrm{~cm}^{-1}$ above the ground state. For this state, the occupied $\pi$ orbitals have the major contributions coming from $\mathrm{P}$ $3 p_{\pi}$, instead of a mixture of metal $3 d_{\pi}$ and $\mathrm{P} 3 p_{\pi}$ orbitals. Actually, as the symmetry and the spin multiplicity of the states change, the coefficients of the atomic orbitals contributing to the molecular orbitals may change as well. We thus suggest that different states might have different bonding schemes, even for the same molecule.

Comparing with the previous ab initio MRCI-SD results of Harrison and co-workers, ${ }^{13}$ their calculated ground state is of ${ }^{2} \Sigma^{+}$symmetry with the fourth valence electron going into the $s d_{\sigma}$ hybrid and the ${ }^{2} \Delta$ state lying $1690 \mathrm{~cm}^{-1}$ above the ${ }^{2} \Sigma^{+}$state. As we have discussed in the preceding section, the difference may be arisen from the inherent shortcomings in the methods used. Jeung ${ }^{27}$ has recently performed MRCI calculations with a more extended basis set and obtained the same ${ }^{2} \Sigma^{+}$ground state. In order to examine whether there is any bias of the B3LYP method for the higher $\Lambda$ state, we have performed B3LYP calculations on the nitride analogue TiN and the isoelectronic diatomic molecule ScS. Both of these two molecules were characterized experimentally to 
TABLE V. Calculated bond lengths $\left(r_{e}\right.$, in $\AA$ ), excitation energies $\left(T_{e}\right.$, in $\left.\mathrm{cm}^{-1}\right)$, dissociation energies $\left(D_{e}\right.$, in $\left.\mathrm{eV}\right)$, harmonic vibrational frequencies $\left(\omega_{e}\right.$, in $\left.\mathrm{cm}^{-1}\right)$, effective nuclear charges $\left(Z_{\text {eff }}\right)$, and dipole moments $\left(\mu_{e}\right.$, in Debye) of VP for several electronic states using the B3LYP method.

\begin{tabular}{lcccccc}
\hline \hline State & $r_{e}$ & $T_{e}$ & $D_{e}{ }^{\mathrm{a}}$ & $\omega_{e}$ & $\mu_{e}$ & $Z_{\mathrm{eff}}{ }^{\mathrm{b}}$ \\
\hline${ }^{3} \Delta$ & 2.140 & 0 & $3.26 / 4.10$ & 390 & 4.24 & 0.42 \\
${ }^{3} \Sigma^{-}$ & 2.168 & 3482 & $2.83 / 3.67$ & 362 & 5.76 & 0.56 \\
${ }^{3} \Pi$ & 2.177 & 9507 & $2.08 / 2.92$ & 392 & 5.84 & 0.56 \\
${ }^{1} \Delta$ & 2.078 & 5514 & $2.57 / 3.42$ & 402 & 4.05 & 0.41 \\
${ }^{1} \Gamma$ & 2.050 & 13545 & $1.58 / 2.42$ & 572 & 5.72 & 0.59 \\
${ }^{1} \Sigma^{-}$ & 2.129 & 17637 & $1.07 / 1.91$ & 416 & 2.85 & 0.28 \\
${ }^{5} \Pi$ & 2.218 & 4878 & $2.65 / 3.50$ & 416 & 4.33 & 0.41 \\
\hline \hline
\end{tabular}

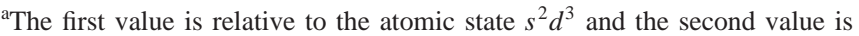
relative to the atomic state $s^{1} d^{4}$.

${ }^{\mathrm{b}} Z_{\mathrm{eff}}=\mu_{e} / r_{e}$.

have a ${ }^{2} \Sigma^{+}$ground state. ${ }^{9,28}$ For both molecules, we predicted the ground state to be ${ }^{2} \Sigma^{+}$, in agreement with experiments and other calculations. Experimental work is required to verify which is the ground state for TiP. As in the case of $\mathrm{ScP}$, the bond lengths from the B3LYP calculations are shorter than the MRCI-SD calculations. ${ }^{13}$ The bond length, vibrational frequency, and dissociation energy (relative to the asymptotic products) of the ${ }^{2} \Sigma^{+}$state calculated by Jeung ${ }^{27}$ are $2.135 \AA$, $491 \mathrm{~cm}^{-1}$, and $2.28 \mathrm{eV}$, respectively. Our results in fact agree quite well with these $a b$ initio values. We have also performed the B3LYP calculations using the same basis set as Jeung ${ }^{27}$ on $\mathrm{ScP}$ and TiP to examine if the results were sensitive to the use of basis sets. We found that the results were more or less the same with the basis set we have used in Sec. II. In fact, there was report indicating that DFT calculation was less dependent on the size of basis set when compared with $a b$ initio methods. ${ }^{29}$ This could be another merit of DFT to be a promising computational tool.

\section{VP}

The spectroscopic parameters for the ground and some excited states of VP are summarized in Table V. The ground state of VP is ${ }^{3} \Delta$. It has a triple bond with two metallocalized, high-spin electrons residing in the $3 d_{\delta}$ and the $s d_{\sigma}$ hybrid orbitals with a bond length of $2.140 \AA$. Singlet coupling the nonbonding $s d_{\sigma}$ electron gives the ${ }^{1} \Delta$ state, which is $5514 \mathrm{~cm}^{-1}$ above the ground state. These two states have similar spectroscopic parameters as they differ only in the spin multiplicity. Exciting the two metal-based, high-spin electrons from the ground state to various higher energy orbitals can generate excited states of VP in which the triple bond is intact. For example, the excitation of the nonbonding $s d_{\sigma}$ electron from the ${ }^{3} \Delta$ state to the $3 d_{\delta}$ orbital gives a ${ }^{3} \Sigma^{-}$ state which lies $3482 \mathrm{~cm}^{-1}$ above the ground state, while moving the electron to the higher-lying $\pi$ orbital gives a ${ }^{3} \Pi$ state with $T_{e}=9507 \mathrm{~cm}^{-1}$. Note that as in the case of TiP, the higher-lying $\pi$ orbital uses both the $3 d_{\pi}-3 p_{\pi}$ and $4 p_{\pi^{-}}-3 p_{\pi}$ overlap. This is due to the same reason as discussed for the ${ }^{2} \Pi$ state of TiP: to minimize the loss in covalent bonding of $\mathrm{P}$ to $\mathrm{V}$ backdonation. Exciting the nonbonding $s d_{\sigma}$ electron from the ${ }^{1} \Delta$ state to the singly occupied $3 d_{\delta}$ orbital gives rise to a ${ }^{1} \Gamma$ state which lies at a much higher
TABLE VI. Calculated bond lengths $\left(r_{e}\right.$, in $\AA$ ), excitation energies $\left(T_{e}\right.$, in $\left.\mathrm{cm}^{-1}\right)$, dissociation energies $\left(D_{e}\right.$, in $\left.\mathrm{eV}\right)$, harmonic vibrational frequencies $\left(\omega_{e}\right.$, in $\left.\mathrm{cm}^{-1}\right)$, effective nuclear charges $\left(Z_{\text {eff }}\right)$, and dipole moments $\left(\mu_{e}\right.$, in Debye) of CrP for several electronic states using the B3LYP method.

\begin{tabular}{lcccccc}
\hline \hline State & $r_{e}$ & $T_{e}$ & $D_{e}{ }^{\mathrm{a}}$ & $\omega_{e}$ & $\mu_{e}$ & $Z_{\mathrm{eff}}{ }^{\mathrm{b}}$ \\
\hline${ }^{4} \Sigma^{-}$ & 2.200 & 0 & $3.63 / 2.21$ & 351 & 4.02 & 0.38 \\
${ }^{4} \Pi$ & 2.164 & 11184 & $2.24 / 0.82$ & 394 & 5.90 & 0.57 \\
${ }^{2} \Sigma^{-}$ & 2.134 & 10614 & $2.31 / 0.89$ & 352 & 3.36 & 0.33 \\
${ }^{6} \Sigma^{-}$ & 2.327 & 5617 & $2.93 / 1.51$ & 327 & 4.17 & 0.38 \\
\hline \hline
\end{tabular}

${ }^{a}$ The first value is relative to the atomic state $s^{2} d^{4}$ and the second value is relative to the atomic state $s^{1} d^{5}$.

${ }^{\mathrm{b}} Z_{\mathrm{eff}}=\mu_{e} / r_{e}$.

energy of $13544 \mathrm{~cm}^{-1}$. High axial angular momentum states generally lie high in energy since they are not correlated to the neutral dissociation limit. If the bonding $s d_{\sigma}$ electron is excited to the empty $3 d_{\delta}$ orbital from the ${ }^{1} \Delta$ state, it will give rise to a ${ }^{1} \Sigma^{-}$state, lying $17637 \mathrm{~cm}^{-1}$ above the ground state, even higher than the ${ }^{1} \Gamma$ state. It is due to the fact that the electron is now excited from a bonding orbital. Moving the $\pi$-bonding electron from the ${ }^{3} \Delta$ state and uncoupling it to the $3 d_{\delta}$ orbital gives rise to a ${ }^{5} \Pi$ state, lying $4878 \mathrm{~cm}^{-1}$ above the ground state.

\section{CrP}

Table VI gives the spectroscopic parameters for the ground and some excited states of $\mathrm{CrP}$. The ground state of $\mathrm{CrP}$ is a ${ }^{4} \Sigma^{-}$state with a bond length $2.200 \AA$. It may be thought of as having a triple bond and three high-spin metal localized electrons in the nonbonding $3 d_{\delta}$ and $s d_{\sigma}$ hybrid orbitals. The first excited state of $\mathrm{CrP}$ of the same multiplicity can be obtained by exciting the unpaired $\sigma$ electron in the ${ }^{4} \Sigma^{-}$state to the higher-lying $\pi$ orbital, giving a ${ }^{4} \Pi$ state. As can be seen from Table VI, there is a large energy gap between these two states $\left(11184 \mathrm{~cm}^{-1}\right)$. This is because, in contrast to the ground state, the in situ valence atomic state of $\mathrm{Cr}$ is no longer solely of $s^{1} d^{5}$, which is of exceptional stability, but a mixture of $s^{1} d^{5}$ and $d^{6}$ atomic states. The latter state is very high lying and hence making the covalent contribution to the bonding decreases. Therefore, there is an increase in the net charge on $\mathrm{Cr}$ relative to the ground state. To minimize the loss of the covalent interaction, there is a mixing between the $3 d_{\pi}$ and the $4 p_{\pi}$ orbitals such that excess charge on $\mathrm{P}$ can be donated to $\mathrm{Cr}$ through the $4 p_{\pi}-3 p_{\pi}$ overlap. Therefore, there is an increase in the $4 p$ population in the ${ }^{4} \Pi$ state. Singlet coupling of the nonbonding $s d_{\sigma}$ electron of the ${ }^{4} \Sigma^{-}$state resulted in the ${ }^{2} \Sigma^{-}$state, which lies $10614 \mathrm{~cm}^{-1}$ above the ground state. One may be surprised about such a huge difference between the two states, which differs only in the spin multiplicity (because $T_{e}$ will be the pairing energy). Such a difference may be attributed to the fact that when the electron is moving from the $\alpha$ to the corresponding $\beta$ spin-orbitals, the coefficients of the atomic orbitals making up the MO's are not the same.

Uncoupling the $\pi$-bonding electron and exciting it to the higher-lying $\pi$ orbitals from the ${ }^{4} \Sigma^{-}$state gives a ${ }^{6} \Sigma^{-}$state, which is $5617 \mathrm{~cm}^{-1}$ above the ground state. Even though this state has the electron occupying a higher-lying orbital, 
TABLE VII. Calculated bond lengths ( $r_{e}$, in $\AA$ ), excitation energies $\left(T_{e}\right.$, in $\left.\mathrm{cm}^{-1}\right)$, dissociation energies $\left(D_{e}\right.$, in $\left.\mathrm{eV}\right)$, harmonic vibrational frequencies $\left(\omega_{e}\right.$, in $\left.\mathrm{cm}^{-1}\right)$, effective nuclear charges $\left(Z_{\text {eff }}\right)$, and dipole moments $\left(\mu_{e}\right.$, in Debye) of MnP for several electronic states using the B3LYP method.

\begin{tabular}{ccccccc}
\hline \hline State & $r_{e}$ & $T_{e}$ & $D_{e}{ }^{\mathrm{a}}$ & $\omega_{e}$ & $\mu_{e}$ & $Z_{\text {eff }}$ \\
\hline${ }^{5} \Pi$ & 2.158 & 0 & 1.56 & 406 & 4.40 & 0.43 \\
${ }^{3} \Sigma^{-}$ & 2.217 & 2112 & 1.30 & 292 & 2.07 & 0.20 \\
${ }^{3} \Pi$ & 2.191 & 7005 & 0.69 & 351 & 3.69 & 0.35 \\
${ }^{1} \Sigma^{-}$ & 2.288 & 19926 & $-0.92^{\mathrm{c}}$ & 283 & 0.01 & 0.00 \\
\hline \hline
\end{tabular}

${ }^{a}$ Reordering orbitals of the atomic state $s^{2} d^{5}$ did not give other states of the same multiplicity. They all converged to the same state.

${ }^{\mathrm{b}} Z_{\text {eff }}=\mu_{e} / r_{e}$.

${ }^{\mathrm{c}}$ See text.

the in situ valence atomic state is still mainly of the stable $s^{1} d^{5}$ state. It is because the $\beta$ electron in the $\pi$ bonding orbital, which is composed of $\mathrm{P} 3 p_{\pi}$ orbital is now moving to the $\alpha$ orbital which is also mainly composed of $\mathrm{P} 3 p_{\pi}$ orbital, with some mixing of $\mathrm{Cr} 3 d_{\pi}$ orbitals. Hence, this state actually lies lower in energy compared with the two previous excited states.

\section{E. MnP}

The spectroscopic parameters for the ground and some excited states of MnP are given in Table VII. The lowest energy state of MnP predicted from the B3LYP method is of ${ }^{5} \Pi$ symmetry with a bond length of $2.155 \AA$. It may be viewed as consisting of a triple bond with the unpaired electrons residing in the nonbonding $s d_{\sigma}$ and $3 d_{\delta}$ and the antibonding $\pi$ orbital. The nonbonding $\sigma$ orbital actually does not arise only from $s d_{\sigma}$ hybridization, but also $4 s p_{\sigma}$. This may be due to the fact that the $s^{1} p^{1} d^{5}$ configuration is about the same energy as $s^{1} d^{6}$, in contrast to all other TM where the $s^{1} p^{1} d^{n}$ state is much higher in energy than both the $s^{2} d^{n}$ and $s^{1} d^{n+1}$ states. ${ }^{30}$ Hence, there is an increase in $4 p$ population. The next higher energy state calculated is ${ }^{3} \Sigma^{-}$, which is formed from the $\mathrm{Mn}$ ground $s^{2} d^{5}$ configuration. It has a triple bond with two valence electrons doubly occupying the $s d_{\sigma}$ hybrid and two valence electrons singly occupying the $3 d_{\delta}$ orbitals. It is calculated to be $2112 \mathrm{~cm}^{-1}$ above the ground state. Even though this state can be made from the ground atomic state of $\mathrm{Mn}$, there is a large loss in $d-d$ exchange energy by spin pairing in this state. Hence, the high spin ${ }^{5} \Pi$ state is favored. Upon excitation of the $s d_{\sigma}$ hybrid electron from the ${ }^{3} \Sigma^{-}$state to the virtual antibonding $\pi$ orbital gives a ${ }^{3} \Pi$ state and it lies $7005 \mathrm{~cm}^{-1}$ above the ground state. Moving the electron from the nonbonding $s d_{\sigma}$ hybrid orbital of ${ }^{3} \Sigma^{-}$state and singlet coupling it to the antibonding $\sigma$ orbital gives the ${ }^{1} \Sigma^{-}$state, which is $19926 \mathrm{~cm}^{-1}$ above the ground state. From the Mulliken population analysis, the ${ }^{1} \Sigma^{-}$state has considerable contributions from the $p$ orbitals, which suggests that this state is arisen from a mixture of the $s^{2} d^{5}$ and the $s^{1} p^{1} d^{5}$ atomic states. Since the $s^{1} p^{1} d^{5}$ state lies roughly $2 \mathrm{eV}$ above the $s^{2} d^{5}$ ground atomic state, the negative dissociation energy indicates that the ${ }^{1} \Sigma^{-}$state probably dissociates to the $s^{1} p^{1} d^{5}$ atomic state and the dissociation energy is still positive relative to the ground atomic state. The antibonding $\sigma$ orbital involves a mixture of $\mathrm{Mn}$
TABLE VIII. Calculated bond lengths $\left(r_{e}\right.$, in $\AA$ ), excitation energies $\left(T_{e}\right.$, in $\left.\mathrm{cm}^{-1}\right)$, dissociation energies $\left(D_{e}\right.$, in $\left.\mathrm{eV}\right)$, harmonic vibrational frequencies $\left(\omega_{e}\right.$, in $\left.\mathrm{cm}^{-1}\right)$, effective nuclear charges $\left(Z_{\text {eff }}\right)$, and dipole moments ( $\mu_{e}$, in Debye) of FeP for several electronic states using the B3LYP method.

\begin{tabular}{lcccccc}
\hline \hline State & $r_{e}$ & $T_{e}$ & $D_{e}{ }^{\mathrm{a}}$ & $\omega_{e}$ & $\mu_{e}$ & $Z_{\mathrm{eff}}{ }^{\mathrm{b}}$ \\
\hline${ }^{6} \Sigma^{+}$ & 2.106 & 0 & $2.07 / 2.59$ & 423 & 3.99 & 0.40 \\
${ }^{6} \Phi$ & 2.229 & 4281 & $1.54 / 2.06$ & 377 & 4.35 & 0.41 \\
${ }^{4} \Phi\left({ }^{4} \Pi\right)$ & 2.103 & 144 & $2.05 / 2.58$ & 413 & 3.62 & 0.36 \\
${ }^{4} \Pi$ & 2.109 & 1315 & $1.91 / 2.43$ & 421 & 4.03 & 0.40 \\
${ }^{2} \Delta$ & 2.150 & 445 & $2.02 / 2.54$ & 347 & 2.75 & 0.27 \\
${ }^{2} \Phi\left({ }^{2} \Pi\right)$ & 2.179 & 1938 & $1.83 / 2.35$ & 343 & 3.14 & 0.30 \\
${ }^{2} \Pi$ & 2.043 & 9019 & $0.95 / 1.47$ & 445 & 4.04 & 0.42 \\
\hline \hline
\end{tabular}

${ }^{a}$ The first value is relative to the atomic state $s^{2} d^{6}$ and the second value is relative to the atomic state $s^{1} d^{7}$.

${ }^{\mathrm{b}} Z_{\mathrm{eff}}=\mu_{e} / r_{e}$.

$4 s, 4 p_{\sigma}$, and $\mathrm{P} 3 p_{\sigma}$ orbitals. As we move across the TM series, not only the $4 s$ and $3 d$ energy decrease, but also the $4 p$ energy, such that for $\mathrm{Mn}$, the $4 p$ energy is close enough to the $\mathrm{P} 3 p$ energy that the antibonding $\sigma$ orbital has a small contribution coming from Mn $4 p_{\sigma}$ orbital. ${ }^{30}$ Thus the metal $4 p$ population increase can be explained.

\section{F. FeP}

The spectroscopic parameters for the ground and some excited states of FeP are presented in Table VIII. The ground state of FeP is calculated to be ${ }^{6} \Sigma^{+}$symmetry with a bond length of $2.106 \AA$. This may be thought of having a double bond with the five valence electrons singly occupying the metal nonbonding $s d_{\sigma}$ hybrid, the $3 d_{\delta}$, and the antibonding $\pi$ orbitals. This state has the in situ valence atomic state arisen from $s^{1} d^{7}$ configuration. As in $\mathrm{MnP}$, the antibonding $\pi$ orbitals have contributions coming not only from $\mathrm{P} 3 p_{\pi}$, but also a small contribution from $\mathrm{Fe} 3 d_{\pi}$ and $4 p_{\pi}$ obitals. This may be due to the continuous fall in $4 p$ energy across the TM series that it has a better match in energy with $\mathrm{P} 3 p$ orbitals. Therefore, there are some populations in the $4 p$ orbitals. The next higher energy state calculated is the ${ }^{4} \Phi$ state (with a small mixing of the ${ }^{4} \Pi$ state), which lies only $144 \mathrm{~cm}^{-1}$ above the ${ }^{6} \Sigma^{+}$state. This state can be thought of consisting of a triple bond with two (one) of the remaining electrons going into the $s d_{\sigma}$ hybrid, two (three) of them going into the $3 d_{\delta}$ orbitals, and the final one going into the antibonding $\pi$ orbitals. It is hard to visualize this state as an electron promoting from the calculated ground state to other orbitals since it is the antibonding $\pi$ electron moving into the nonbonding orbitals, which should make this state lower in energy. We have looked into the population of the MO's formed from the valence orbitals for these two states. We found that the metal and $\mathrm{P}$ valence orbitals actually mix differently in these two states. For example, the bonding $\pi$ orbital has the contributions coming mainly from $\mathrm{Fe} 3 d_{\pi}$ in ${ }^{6} \Sigma^{+}$state; however, in ${ }^{4} \Phi\left({ }^{4} \Pi\right)$ state, the $\beta$ spin-orbital main contributions come from $\mathrm{P} 3 p_{\pi}$. This may be the reason why even though the ${ }^{6} \Sigma^{+}$state has two electrons going into the antibonding $\pi$ orbitals, it is still of lower energy. Moreover, again according to Hund's rule, high spin states should be favored. Therefore, ${ }^{6} \Sigma^{+}$state is very likely to be 
the ground state. The second higher energy state calculated is of ${ }^{2} \Delta$ symmetry, which consists of a triple bond with the five valence electrons occupying the nonbonding $s d_{\sigma}$ and $3 d_{\delta}$ orbitals. This state lies only $445 \mathrm{~cm}^{-1}$ above the ground state. From the population analysis, the in situ valence atomic state is most likely to be $s^{1} d^{7}$. As is discussed above, the different mixings of the atomic orbitals and the low spin multiplicity may be the reason why even though it has no electron occupying the antibonding orbitals, it is still of higher energy. However, since these three states are so close in energy (only hundreds of $\mathrm{cm}^{-1}$ difference), we cannot conclude that ${ }^{6} \Sigma^{+}$must be the ground state. In the nitride analogue, it has been proposed that ${ }^{2} \Delta$ and ${ }^{4} \Pi$ states to be the ground states. ${ }^{9}$ It would need further studies to confirm the ground state for FeP.

Excitation of the $s d_{\sigma}$ hybrid electron of the ${ }^{2} \Delta$ state to the antibonding $\pi$ orbital gives rise to the ${ }^{2} \Phi$ and ${ }^{2} \Pi$ states. The ${ }^{2} \Phi$ state is calculated to be $1938 \mathrm{~cm}^{-1}$ above the ${ }^{2} \Delta$ state, with a small mixings of a ${ }^{2} \Pi$ state (this $\Pi$ state arises from promoting a $3 d_{\delta}$ electron to the antibonding $\pi$ orbital from the ${ }^{2} \Delta$ state), while the ${ }^{2} \Pi$ state is lying $9019 \mathrm{~cm}^{-1}$ above the ${ }^{2} \Delta$ state. One may be surprised why such a large difference is observed since they only differ in the sign of the antibonding orbital occupied ( $\pi_{-}$and $\pi_{+}$components, respectively). This is because, from the population of these two orbitals in the respective state, ${ }^{2} \Phi$ state has the $\pi_{-}$composed of Fe $3 d_{\pi}$ and P $3 p_{\pi}$, while the ${ }^{2} \Pi$ state has the $\pi_{+}$ made of mainly $\mathrm{P} 3 p_{\pi}$ (with a small contribution from $\mathrm{Fe}$ $3 d_{\pi}$ and $\left.4 p_{\pi}\right)$. Hence, they would not be of the same energy. Moreover, these two states also have different mixings of the AO's to give the MO's. Therefore, it may not be appropriate to say simply that the excited states are arisen from promoting the electron from one MO to another MO because of large orbital relaxation. Uncoupling the nonbonding $s d_{\sigma}$ electron of the ${ }^{2} \Pi$ state gives rise to the ${ }^{4} \Pi$ state, which is $1315 \mathrm{~cm}^{-1}$ above the ground state. These two states are separated by $\sim 7700 \mathrm{~cm}^{-1}$ not only because of the difference in spin multiplicity, but also there is a slight difference in the AO composition in the nonbonding $s d_{\sigma}$ orbital: the ${ }^{4} \Pi$ state has a larger $4 s$ character than the ${ }^{2} \Pi$ state. If an electron is excited from the bonding $\pi$ orbitals of the ${ }^{6} \Sigma^{+}$state to the antibonding $\pi$ orbitals, it gives rise to the ${ }^{6} \Phi$ state. This state lies $4281 \mathrm{~cm}^{-1}$ above the ground state. Here, the electron configuration change includes an orbital substitution from the $\mathrm{Fe}$ character to the $\mathrm{P}$ character, accompanied by a net electron charge transfer from $\mathrm{Fe}$ to $\mathrm{P}$. Thus, there is an increase in the net charge relative to the ground state.

\section{G. CoP}

Table IX lists the spectroscopic parameters for the ground and some excited states of CoP. The ground state of CoP has ${ }^{5} \Delta$ symmetry. From population analysis, it can be seen that the in situ valence atomic configuration for this state is $\operatorname{Cos} s^{1} d^{8}$. It consists of a double bond of bond length $2.125 \AA$ with three valence electrons going into the $3 d_{\delta}$ orbitals and the remaining three singly occupying the $s d_{\sigma}$ hybrid and the antibonding $\pi$ orbitals. Coupling the nonbonding $3 d_{\delta}$ electron gives rise to the ${ }^{3} \Delta$ state which is $6727 \mathrm{~cm}^{-1}$ above the ground state. This difference in energy
TABLE IX. Calculated bond lengths $\left(r_{e}\right.$, in $\AA$ ), excitation energies $\left(T_{e}\right.$, in $\left.\mathrm{cm}^{-1}\right)$, dissociation energies $\left(D_{e}\right.$, in $\left.\mathrm{eV}\right)$, harmonic vibrational frequencies $\left(\omega_{e}\right.$, in $\left.\mathrm{cm}^{-1}\right)$, effective nuclear charges $\left(Z_{\text {eff }}\right)$, and dipole moments $\left(\mu_{e}\right.$, in Debye) of CoP for several electronic states using the B3LYP method.

\begin{tabular}{lcccccc}
\hline \hline State & $r_{e}$ & $T_{e}$ & $D_{e}{ }^{\mathrm{a}}$ & $\omega_{e}$ & $\mu_{e}$ & $Z_{\mathrm{eff}}{ }^{\mathrm{b}}$ \\
\hline${ }^{5} \Delta$ & 2.125 & 0 & $2.29 / 2.23$ & 378 & 3.68 & 0.36 \\
${ }^{3} \Phi$ & 2.085 & 651 & $2.21 / 2.15$ & 393 & 3.10 & 0.31 \\
${ }^{3} \Delta$ & 2.137 & 6727 & $1.46 / 1.40$ & 353 & 3.48 & 0.34 \\
${ }^{3} \Pi$ & 2.038 & 11227 & $0.90 / 0.84$ & 378 & 3.26 & 0.34 \\
${ }^{1} \Phi\left({ }^{1} \Pi\right)$ & 2.148 & 645 & $2.21 / 2.15$ & 350 & 2.88 & 0.28 \\
${ }^{1} \Phi$ & 2.127 & 2095 & $2.03 / 1.97$ & 359 & 2.67 & 0.26 \\
${ }^{1} \Sigma^{+}$ & 1.909 & 10392 & $1.00 / 0.94$ & 587 & 2.22 & 0.24 \\
\hline \hline
\end{tabular}

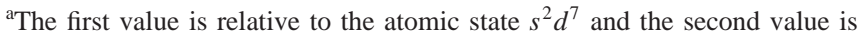
relative to the atomic state $s^{1} d^{8}$.

${ }^{\mathrm{b}} Z_{\mathrm{eff}}=\mu_{e} / r_{e}$.

between the two states is due to the difference in spin multiplicity as their spectroscopic parameters differ only slightly. Exciting the $\pi$-bonding electron in the ${ }^{5} \Delta$ state to the nonbonding $s d_{\sigma}$ hybrid and coupling the two antibonding $\pi$ electrons gives rise to a ${ }^{3} \Phi$ state at an energy $651 \mathrm{~cm}^{-1}$ above the ground state. If the bonding electron goes into the nonbonding $3 d_{\delta}$ orbital instead, it will give rise to a ${ }^{3} \Pi$ state lying $11227 \mathrm{~cm}^{-1}$ above the ground state. If we have all the electrons singlet coupled in the ${ }^{3} \Phi$ state, it will give rise to a ${ }^{1} \Phi$ state, which is $2095 \mathrm{~cm}^{-1}$ above the ground state. If this state is mixed with a ${ }^{1} \Pi$ state (this state arises from fully occupying the $3 d_{\delta}$ instead of the $s d_{\sigma}$ nonbonding hybrid as in the ${ }^{1} \Phi$ state), it significantly lowers its energy to $645 \mathrm{~cm}^{-1}$ above the ground state. Excitation of all the $\pi$-bonding electrons to the nonbonding $3 d_{\delta}$ and higher-lying $\pi$ orbitals from the ${ }^{3} \Phi$ state results in a ${ }^{1} \Sigma^{+}$state which is $10392 \mathrm{~cm}^{-1}$ above the ground state. It may be surprising why the ${ }^{1} \Sigma^{+}$state is lower in energy than ${ }^{3} \Pi$ state, which has more bonding electrons. This is because the higher-lying $\pi$ orbitals in the ${ }^{1} \Sigma^{+}$state is mainly of $\mathrm{P} 3 p$ character. Hence, it has a strong bonding between the $\mathrm{P} 3 p_{\pi}$ orbitals and results in a significant decrease in bond length and a slightly lower energy than the ${ }^{3} \Pi$ state.

\section{H. NiP}

The spectroscopic parameters for the ground and some excited states of NiP are given in Table X. The lowest energy state of NiP calculated is ${ }^{4} \Delta$ with a bond length of $2.127 \AA$.

TABLE X. Calculated bond lengths ( $r_{e}$, in $\AA$ ), excitation energies $\left(T_{e}\right.$, in $\left.\mathrm{cm}^{-1}\right)$, dissociation energies $\left(D_{e}\right.$, in $\left.\mathrm{eV}\right)$, harmonic vibrational frequencies $\left(\omega_{e}\right.$, in $\left.\mathrm{cm}^{-1}\right)$, effective nuclear charges $\left(Z_{\text {eff }}\right)$, and dipole moments $\left(\mu_{e}\right.$, in Debye) of NiP for several electronic states using the B3LYP method.

\begin{tabular}{lcccccr}
\hline \hline State & $r_{e}$ & $T_{e}$ & $D_{e}{ }^{\mathrm{a}}$ & $\omega_{e}$ & $\mu_{e}$ & \multicolumn{1}{c}{$Z_{\text {eff }}{ }^{\mathrm{b}}$} \\
\hline${ }^{4} \Delta$ & 2.127 & 0 & $2.77 / 2.32$ & 391 & 2.86 & 0.28 \\
${ }^{4} \Sigma^{-}$ & 2.141 & 1721 & $2.56 / 2.11$ & 347 & 3.36 & 0.33 \\
${ }^{2} \Delta$ & 2.130 & 2284 & $2.49 / 2.04$ & 389 & 2.85 & 0.28 \\
${ }^{2} \Pi$ & 2.281 & 18077 & $0.53 / 0.07$ & 304 & -0.49 & -0.05 \\
${ }^{6} \Delta$ & 2.353 & 13990 & $1.03 / 0.58$ & 250 & 0.66 & 0.06 \\
\hline \hline
\end{tabular}

${ }^{a}$ The first value is relative to the atomic state $s^{2} d^{8}$ and the second value is relative to the atomic state $s^{1} d^{9}$.

${ }^{\mathrm{b}} Z_{\mathrm{eff}}=\mu_{e} / r_{e}$. 
TABLE XI. Calculated bond lengths $\left(r_{e}\right.$, in $\AA$ ), excitation energies $\left(T_{e}\right.$, in $\left.\mathrm{cm}^{-1}\right)$, dissociation energies $\left(D_{e}\right.$, in $\left.\mathrm{eV}\right)$, harmonic vibrational frequencies $\left(\omega_{e}\right.$, in $\left.\mathrm{cm}^{-1}\right)$, effective nuclear charges $\left(Z_{\text {eff }}\right)$, and dipole moments $\left(\mu_{e}\right.$, in Debye) of $\mathrm{CuP}$ for several electronic states using the B3LYP method.

\begin{tabular}{lcccccc}
\hline \hline State & $r_{e}$ & $T_{e}$ & $D_{e}{ }^{\mathrm{a}}$ & $\omega_{e}$ & $\mu_{e}$ & \multicolumn{1}{c}{$Z_{\text {eff }}{ }^{\mathrm{b}}$} \\
\hline${ }^{3} \Sigma^{-}$ & 2.160 & 0 & 2.13 & 358 & 2.82 & 0.27 \\
${ }^{3} \Pi$ & 2.135 & 13658 & 0.43 & 355 & 4.08 & 0.40 \\
${ }^{1} \Sigma^{-}$ & 2.151 & 1966 & 1.89 & 365 & 2.87 & 0.28 \\
${ }^{1} \Delta$ & 2.123 & 9164 & 0.99 & 388 & 2.96 & 0.29 \\
${ }^{5} \Sigma^{-}$ & 2.420 & 17311 & $-0.02^{\mathrm{c}}$ & 160 & -0.89 & -0.08 \\
\hline \hline
\end{tabular}

${ }^{\text {a Reordering the orbitals of the atomic state } s^{1} d^{10} \text { did not give other states of }}$ the same multiplicity. They all converged to the same state.

${ }^{\mathrm{b}} Z_{\mathrm{eff}}=\mu_{e} / r_{e}$.

${ }^{\mathrm{c}}$ Presence of barrier.

The in situ valence atomic configuration for this state is $s^{1} d^{9}$ configuration with the $d$ hole in the $\delta$ symmetry. Singlet coupling of the two electrons occupying the higher-lying $\pi$ orbitals results in a ${ }^{2} \Delta$ state, which lies $2284 \mathrm{~cm}^{-1}$ above the ground state. Since the two states differ only in the spin multiplicity, their spectroscopic parameters are very close to each other. Uncoupling the nonbonding $s d_{\sigma}$ electron in the ground state and promoting it to the antibonding $\sigma$ orbital gives rise to a ${ }^{6} \Delta$ state. This state lies $13990 \mathrm{~cm}^{-1}$ above the ground state and because the electron moves from a nonbonding orbital to an antibonding orbital, the bond length increases. From population analysis, it can be seen that there is significant population in the $4 p_{\sigma}$ orbital, indicating that besides $s d_{\sigma}$ hybridization, there is also $4 s p_{\sigma}$ hybridization for bonding in NiP. As discussed in the section of MnP, it is probable that as the energy of $4 p$ orbitals continue to fall across the TM series the $4 p-3 p$ overlap becomes more favorable for bonding. On the other hand, if the $s d_{\sigma}$ electron in the ground state moves to the metal $3 d_{\delta}$ orbital, it gives rise to a ${ }^{4} \Sigma^{-}$state lying $1721 \mathrm{~cm}^{-1}$ above the ground state. Since the electron is transferred among the nonbonding orbitals, the bond lengths do not differ much. If an electron is excited from the $\pi$-bonding orbital in the ${ }^{2} \Delta$ state to the antibonding $\sigma$ orbital, it gives a ${ }^{2} \Pi$ state, which is $18077 \mathrm{~cm}^{-1}$ above the ground state. This state arises not only from $s^{2} d^{8}$ and $s^{1} d^{9}$ configurations, but also $s^{1} p^{1} d^{8}$ configuration, as in the case of the ${ }^{6} \Delta$ state. Since the $s^{1} p^{1} d^{8}$ configuration is high-lying relative to the ground atomic state, the states arising from this configuration are rather high in energy. Moreover, the ${ }^{2} \Pi$ state actually has a net negative charge on $\mathrm{Ni}$, instead of the more electronegative $\mathrm{P}$. This may be due to the excitation of the essentially $\mathrm{Ni} 3 d_{\pi}$ electron, leaving a hole for $\mathrm{P}$ to have charge transfer from $\mathrm{P}$ to $\mathrm{Ni}$.

\section{CuP}

The spectroscopic parameters for the ground and some excited states of CuP are given in Table XI. The ground state of CuP calculated is mainly arisen from the ground $s^{1} d^{10}$ configuration, with a small contribution coming from the $s^{2} d^{9}$ and $s^{1} p^{1} d^{9}$ configurations, forming a ${ }^{3} \Sigma^{-}$state. It has a bond length of $2.160 \AA$ and consists of a double bond with all the nonbonding orbitals fully filled. Singlet coupling of the high-lying $\pi$ electrons gives a ${ }^{1} \Sigma^{-}$state lying $1966 \mathrm{~cm}^{-1}$ above the ground state. These two states differ mainly in the spin multiplicity and hence they have very similar spectroscopic parameters. When the nonbonding $s d_{\sigma}$ electron in the ground state is excited to the higher-lying $\pi$ orbital, it gives rise to a ${ }^{3} \Pi$ state which lies $13658 \mathrm{~cm}^{-1}$ above the ground state. Since the higher-lying $\pi$ orbitals are mainly of $\mathrm{P}$ character, there is net negative charge transferred to $\mathrm{P}$, and this is the only state calculated to have negative charge on $\mathrm{P}$. This state is much higher in energy than the ground state since this excited state is arisen not only from the very stable ground $s^{1} d^{10}$ configuration, but also some contributions from the high-lying $s^{1} p^{1} d^{9}$ configuration. Though this atomic state is also involved in the ground state, it is not as much as in this excited state. If the high-lying $\pi$ electron in the ${ }^{1} \Sigma^{-}$state now sits in the same space, say $\pi_{+}$, the state resulted will be of ${ }^{1} \Delta$ symmetry which lies $9164 \mathrm{~cm}^{-1}$ above the ground state. Such difference in energy between these two singlet states is due to the fact that the ${ }^{1} \Delta$ state has the two electron closer together which is unfavorable according to the Hund's rule and hence, it has a higher energy. Uncoupling the $s d_{\sigma}$ electron in the ${ }^{3} \Sigma^{-}$state and exciting it to the antibonding $\sigma$ orbital gives rise to the ${ }^{5} \Sigma^{-}$ state. This state is $17311 \mathrm{~cm}^{-1}$ above the ground state. Such a large energy gap may indicate that this $\sigma$ orbital has a strong antibonding character.

\section{CONCLUSIONS}

We have performed B3LYP calculations with an extended basis set on the first-row TM phosphides. We found that the ground states of these phosphides followed those of the isoelectronic sulfides (except TiP, where a ground state of ${ }^{2} \Delta$ is predicted from our B3LYP calculations, but a ${ }^{2} \Sigma^{+}$ state for $\mathrm{ScS}) .^{31}$ In addition, the covalent character of these phosphides increases across the series from $\mathrm{Sc}$ to $\mathrm{Cu}$ (with a slight rise at $\mathrm{Mn}$ ), in accordance with the electronegativity difference. Electronic states with different spin multiplicities are well separated for the early and late phosphides, but not for those in the middle, in particular FeP and CoP. Compounds formed by these TM's are in many cases difficult to describe accurately as one has to balance the $d-d$ exchange energy loss against the energy gain in bond formation. This quasidegeneracy makes it difficult to assign using theoretical approach unambiguously which state should be the ground state, so all these low-lying states are possible candidates to be the ground state. It would need further experimental and theoretical studies to confirm the ground state, in particular those for the TiP, FeP, and CoP.

\section{ACKNOWLEDGMENT}

One of the authors (A.S.C.C.) would like to thank the French Ministry of University and Research for a visiting professorship to the Universite de Provence.

\footnotetext{
${ }^{1}$ M. Wojiechowska, J. Haber, S. Lomnicki, and J. Stoch, J. Mol. Catal. A: Chem. 141, 155 (1999).

${ }^{2}$ C. N. R. Rao, Annu. Rev. Phys. Chem. 40, 291 (1989).

${ }^{3}$ W. Welter, Jr., Science 155, 155 (1967).

${ }^{4}$ N. M. White and R. F. Wing, Astrophys. J. 222, 209 (1978).
} 
${ }^{5}$ A. Veillard, Chem. Rev. 91, 743 (1991).

${ }^{6}$ N. Koga and K. Morokuma, Chem. Rev. 91, 823 (1991).

${ }^{7}$ A. J. Merer, Annu. Rev. Phys. Chem. 40, 407 (1989).

${ }^{8}$ M. Hargittai, Chem. Rev. 100, 2233 (2000).

${ }^{9}$ K. Huber and G. Herzberg, Molecular Spectra and Molecular Structure (van Nostrand Reinhold, New York, 1979), Vol. V.

${ }^{10}$ B. Aronsson, T. Landstrom, and S. Rurdquist, Borides, Silicides and Phosphides (Wiley, New York, 1965).

${ }^{11}$ P. E. M. Siegbahn, Adv. Chem. Phys. 93, 1333 (1996).

${ }^{12}$ F. Tientega and J. F. Harrison, Chem. Phys. Lett. 223, 202 (1994).

${ }^{13}$ V.-A. Glezakou, A. Mavridis, and J. F. Harrison, J. Phys. Chem. 100, 13971 (1996).

${ }^{14}$ H. Gomez, T. R. Taylor, Y. Zhao, and D. M. Neumark, J. Chem. Phys. 117, 8644 (2002).

${ }^{15}$ A. D. Becke, J. Chem. Phys. 98, 5648 (1993).

${ }^{16}$ P. J. Stevens, J. F. Devlin, C. F. Chabalowski, and M. J. Frisch, J. Phys. Chem. 98, 11623 (1994).

${ }^{17}$ G. S.-M. Tong and A. S.-C. Cheung, J. Phys. Chem. A 106, 11637 (2002).

${ }^{18}$ All basis sets are taken from the web site of the EMSL Basis Set Library. http://www.emsl.pnl.gov:2080/forms/basisform.html

${ }^{19}$ M. J. Frisch, G. W. Trucks, H. B. Schlegel et al., GAUSSIAN 98, Revision A.4, Gaussian, Inc., Pittsburgh, PA, 1998.

${ }^{20}$ R. Stowasser and R. Hoffmann, J. Am. Chem. Soc. 121, 3414 (1999).
${ }^{21}$ T. Kar, J. G. Ángyán, and A. B. Sannigraphi, J. Phys. Chem. A 104, 9953 (2000).

${ }^{22}$ I. N. Levine, Quantum Chemistry (Prentice Hall, New Jersey, 1991), p. 289.

${ }^{23}$ S. Yanagisawa, T. Tsuneda, and K. Hirao, J. Chem. Phys. 112, 545 (2000).

${ }^{24}$ The calculated ${ }^{3} \mathrm{~F}-{ }^{5} \mathrm{~F}$ separation of Ti atom using the B3LYP method was $0.25 \mathrm{eV}$, which is lower than the experimental value of $0.81 \mathrm{eV}$ (Ref. 25).

${ }^{25}$ C. E. Moore, Ionization Potential and Ionization Limits from the Analysis of Optical Spectra, Natl. Bur. Stand. Ref. Data Ser., Natl. Bur. Stand. (U.S.) Circ. No. 34 (U.S. GPO, Washington, D.C., 1970).

${ }^{26}$ See EPAPS Document No. E-JCPSA6-118-305320 for the population analysis of the nine transition metal phosphides. A direct link to this document may be found in the online article's HTML reference section. The document may also be reached via the EPAPS homepage (http:// www.aip.org/pubservs/epaps.html) or from ftp.aip.org in the directory lepaps/. See the EPAPS homepage for more information.

${ }^{27} \mathrm{G}$. H. Jeung (unpublished results).

${ }^{28}$ T. M. Dunn, L. K. Hanson, and R. A. Rubinson, Can. J. Phys. 48, 1657 (1970).

${ }^{29}$ S. Tsuzuki and H. P. Ldüthi, J. Chem. Phys. 114, 3949 (2001).

${ }^{30}$ J. F. Harrison, Chem. Rev. 100, 679 (2000).

${ }^{31}$ C. W. Bauschlicher, Jr. and P. Maitre, Theor. Chim. Acta 90, 189 (1995). 